\title{
Factors affecting emergency medical dispatchers' decision-making: a qualitative study
}

This article was published in the following Dove Press journal: Journal of Multidisciplinary Healthcare

\author{
Seyyed Mohammad Reza \\ Hosseini' \\ Mohammadreza Maleki' \\ Hasan Abolghasem Gorji' \\ Davoud \\ Khorasani-Zavareh ${ }^{2-4}$ \\ Masoud Roudbari ${ }^{5}$ \\ 'Department of Health Services \\ Management, School of Health \\ Management and Information \\ Sciences, Iran University of Medical \\ Sciences, Tehran, Iran; ${ }^{2}$ Safety \\ Promotion and Injury Prevention \\ Research Center, Shahid Beheshti \\ University of Medical Sciences, \\ Tehran, Iran; ${ }^{3}$ Department of Health \\ in Disaster and Emergency, School \\ of Health, Safety and Environment, \\ Shahid Beheshti University of Medical \\ Sciences, Tehran, Iran; ${ }^{4}$ Department \\ of Clinical Sciences and Education, \\ Karolinska Institute, Södersjukhuset \\ (KI SÖS), Stockholm, Sweden; \\ ${ }^{5}$ Antimicrobial Resistance Research \\ Center, Rasoul-e-Akram Hospital, \\ Department of Biostatistics, School \\ of Public Health, Iran University of \\ Medical Sciences, Tehran, Iran
}

Correspondence: Mohammadreza Maleki Department of Health Services Management, Iran University of Medical Sciences, No 4, Shahid Yasemi Street, Vali-e-Asr Avenue (near Khatam OI Anbia Hospital), Tehran, 19956 I4III, Iran

Tel +989/2 I05 5069

Fax +98 2188793805

Email Maleki.mr@iums.ac.ir
Introduction: Prehospital emergency medical service dispatchers should make prompt and appropriate decisions to save the life of victims. The complexity of timely and reasonable decision-making in life-threatening conditions has driven researchers to investigate varying aspects of the emergency medical dispatch (EMD) process. The purpose of this study was to explore the contributors to appropriate and prompt decision-making among dispatchers.

Methods: A qualitative study through thematic analysis was designed. Data were collected using observation and semistructured interviews with 16 authorities and dispatchers in seven EMDs across Iran.

Results: The study found "responsiveness" as the main category contributing to improved decision-making in EMD. The components introduced in this study for dispatchers' responsiveness consisted of two categories. The first was "personal values" including faith and belief, eagerness to help, service excellence, altruism, respect, and impartiality in clinical judgment. The second was "professional attitudes" resulting from education and experience, including the recognition of emergency as a threat to health, sensitivity in triage, response to all requests for help, care for early warnings, commitment to organizational goals and standards, attention to the emergency medical service social support responsibility, and professional temperance.

Conclusion: In this study, responsiveness was identified as a main category in improving the decision-making process among dispatchers. To attain responsiveness, institutionalization of its values and establishment of EMD-specific professional attitudes in dispatchers should be taken into consideration.

Keywords: emergency medical services, emergency medical dispatch, response time, decisionmaking, prehospital, dispatcher

\section{Introduction}

A prompt prehospital response is conducive to improved prognosis of victims and stands as one of the goals of the emergency medical services (EMSs). ${ }^{1}$ Timely access to EMS at the accident site can reduce the 1 million deaths and 50 million accidentinduced disabilities occurring every year throughout the world. ${ }^{2,3}$

To reduce mortality, it is vital to improve EMS, especially in low- and middleincome countries. ${ }^{4,5}$ However, assessment of the EMS in these countries reflects both a gap from the updated scientific knowledge and the need for better understanding of the shortcomings and solution. ${ }^{5}$ In recent years, EMS in Iran has had some progress in reducing the high incidence of accident-induced and cardiovascular mortality, ${ }^{6}$ although there is still room to develop its process and reach universal standards. ${ }^{7}$ 
Therefore, it is crucial to identify the factors conducive to EMS improvement.

In the Iranian context, the EMS was founded simultaneously in major cities of the country in 1975. It was organized as a government agency with free services for all patients and gradually turned into a nationwide organization covering the whole country. ${ }^{8}$ The Iranian Ministry of Health and Medical Education has been developing proprietary capacity for prehospitalization, triage, and drainage protocols, and has steadily increased the number of ambulances, EMS stations, and airbase ambulances since 2003. ${ }^{9}$ In Iran, dispatchers typically tend to have an associate or a bachelor's degree in medical emergency or nursing. They also usually attend short-term programs on dispatching.

As the key section of the EMS, emergency medical dispatch (EMD) is in charge of receiving and managing requests for assistance. The emergency medical dispatcher is expected to have timely communication with the caller and the ambulance group, and to decide on patient triage and ambulance dispatch. Any wrong decision made by the dispatcher in the dispatch of the ambulance will delay the initiation of care and mitigate subsequent attempts to help the victim..$^{10}$ Of course, the EMD's work environment abounds in challenges and tensions and can affect the quality of the abovementioned measures. ${ }^{11}$

Research suggests limitations in understanding EMD processes, and the study of how EMD operates has been demanded in different cultures and settings. ${ }^{12}$ Despite the importance of time in the initiation of prehospital care, few studies have covered the EMD process and opportunities for improving its time intervals. ${ }^{13}$ Moreover, whereas the qualitative and human aspects of EMD are attractive, only few studies have been conducted on those issues. ${ }^{13}$ Existing investigations generally have a quantitative approach, notwithstanding the fact that the stakeholders' views and attitudes are fundamental to improving quality. ${ }^{14}$ As EMD decisions are made in a short time upon limited information, it is recommended to understand its processes more profoundly. ${ }^{15}$

Knowledge of the factors that influence the prompt and proper decision-making of the dispatcher is necessary to ensure that right decisions are made. In this regard, a study comparing two cities in Iran and Sweden reports that while in the Swedish city, all patients were transferred to the hospital by the EMS with no right for the EMS to refuse transference of victims, in the studied city in Iran, only $36 \%$ of the patients were hospitalized. The author has recommended evaluating the correctness of these decisions. ${ }^{16}$
In this article, we focus on the human factors contributing to the improvement of dispatchers' decision-making, an issue that has not been reviewed before. There seems to be many unanswered questions about the EMD process and the underlying reason for adoption of different actions, which are taken as the research questions in this study: What are the factors influencing decision-making in unpredictable and unexpected EMD situations? How do EMD personnel act in tense conditions? How do they react in complex situations?

The results of this study can fill the gap between quantitative studies and create new attitudes aiming to improve the timely and appropriate decision-making in the EMD process. Our goal in this study is to explore the contributing factors to timely and appropriate decision-making of EMS dispatchers.

\section{Methods}

This qualitative thematic analysis was conducted in Iran in 2016. The data were collected by observation and semistructured interviews. Thematic analysis is a suitable method to understand, analyze, and report themes, where the data are organized and analyzed in a perfect and precise manner, thereby different aspects of the issue of interest are revealed. Thematic analysis is one of the approaches to qualitative studies, which is used to analyze the data and to extract codes and themes from the data. ${ }^{17,18}$

\section{Study setting and sampling}

The study was conducted in seven EMDs based in different cities of Iran with different populations (including the capital city, metropolitan cities, and middle-sized counties). Selected via purposive sampling with maximum variation, the participants comprised 16 people from among officials and dispatches who had a minimum tenure of 2 years. Moreover, in order to get acquainted with various aspects of the EMD environment and decision-making process, the triangulation of observation and interview was employed.

\section{Data collection}

This study was conducted via semistructured interviews with the selected stakeholders. In addition, the data were collected by direct observation and note-taking on the dispatching process using the observer's guide. Interviews continued until the data were saturated. The average time required for each interview was from half an hour to one hour. Before the interview initiated, enough explanation was given about the goals of the study and, while ensuring confidentiality of the data, a written consent form was received from the interviewees. During the interviews, notes were taken and, at the end of 
each interview, the highlighted points and a summary of the participants' responses were restated to confirm the accuracy of the data. The interviews were conducted individually in the workplace of the participants.

\section{Data analysis}

All the interviews were recorded and transcribed verbatim by the investigator. Allocation of sufficient time to data collection, along with frequent examination of the documents, the texts, and the notes provided a profound immersion of the investigator with the data collected. Besides, the researcher identified and withstood his personal ideas and beliefs before and during the process of data collection and analysis in order to prevent from their interference. Data analysis initiated simultaneously with data collection and continued until full saturation of the data. The collected data underwent thematic analysis, and the search for explicit and implicit issues related to the topic under study was carried out by observing and carefully reviewing the relevant activities. This was achieved by segmenting the text into units of meaning and their subsequent summarization whereby 13 codes emerged. Similar and common codes were grouped into two categories, and the main category was formed by integrating the basic meanings in the categories in order to identify the contributors to the decision-making of the dispatchers.

\section{Trustworthiness}

In order to increase the trustworthiness of the study, the following measures were taken. To ensure the credibility of the data, enough time was spent to collect and review the data using different methods such as the use of varying participants in terms of occupational and educational levels. To guarantee transferability, appropriate quotations are used and all stages of the study, including sampling, data collection, and analysis, are described in detail and with clarity. To ensure data dependability, the texts were simultaneously analyzed as the interviews were made, thereby the analysis process was revised and confirmed by research colleagues. Finally, conformability of the data was assured via the confirmation of the interview transcriptions by the participants.

\section{Ethical considerations}

Before the data collection process initiated, ethical permission was obtained from the Ethics Committee of Iran University of Medical Sciences (Project Number: 1393/30). Also, the purpose of the study was explained to the participants whereby they provided informed consent for participation in the study. Meanwhile, all participants were reassured that the interviews were completely confidential and that all audio files would be deleted at the end of the study.

\section{Results}

In this study, the codes related to the factors influencing the decision-making of dispatchers were first aggregated and studied, leaving 13 codes upon elimination of repetitive cases. The set of emerged codes for contributors to decision-making of dispatchers were sorted into the two categories of values (including faith and belief, eagerness to help, service excellence, altruism, respect, and impartiality in clinical judgment) and professional attitudes (including recognition of emergency as any threat to health, sensitivity in triage, response to all requests for help, care for early warnings, commitment to organizational goals and standards, attention to the EMS social support responsibility, and professional temperance as well as a main category, namely, responsiveness [Table 1]).

\section{Personal values and beliefs}

The participants highlighted the role of values and beliefs for dispatchers in their prompt and proper decision-making.

\section{Faith and belief}

The participants considered religious beliefs and values as an incentive for maximum possible assistance and sympathetic

Table I Main category, categories, and codes extracted on contributors to dispatchers' decision-making

\begin{tabular}{|l|l|l|}
\hline Codes & Categories & $\begin{array}{l}\text { Main } \\
\text { category }\end{array}$ \\
\cline { 1 - 1 } Faith and belief & & \\
\cline { 1 - 1 } Eagerness to help & Responsiveness \\
\cline { 1 - 1 } Service excellence & \\
\cline { 1 - 1 } Altruism & \multirow{2}{*}{$\begin{array}{l}\text { Professional } \\
\text { Respect }\end{array}$} & \\
\cline { 1 - 1 } Impartiality in clinical judgment & \\
\cline { 1 - 1 } thecognition of emergency as any & \\
\cline { 1 - 1 } Sensitivity in triage & \\
\cline { 1 - 1 } Response to all requests for help & \\
\cline { 1 - 1 } Care for early warnings & \\
\cline { 1 - 1 } $\begin{array}{l}\text { Commitment to organizational goals } \\
\text { and standards }\end{array}$ & \\
\cline { 1 - 1 } $\begin{array}{l}\text { Attention to the EMS social support } \\
\text { responsibility }\end{array}$ & \\
\cline { 1 - 1 } Professional temperance & \\
\cline { 1 - 2 } & & \\
\hline
\end{tabular}

Abbreviation: EMS, emergency medical service. 
services to patients as they regarded themselves responsible to save patients' lives before God:

Authorities happen in many cases to leave our efforts unrecognized, but I do the best for the patient for the sake of God. [...] When you work with belief, [...] people also understand it.

\section{Eagerness to help}

The participants noted that their work would be tedious and stressful if they had no interest in helping others and that it would be better for them to express this interest through a sincere conversation with the patient. Interestingly, the callers also sensed the enthusiasm in the dispatchers, and thus appreciated and cooperated better with the dispatcher:

I like to help people. [...] Sometimes the patient did not have a good ending, but the patient's companion thanked me because I made my best to help the patient. [Contributor 6]

It seems to me that you should encourage the patient to depict a more precise medical history [...] and make him/ her feel that you are eager to help. [Contributor 4]

\section{Service excellence}

The participants considered the provision of high-quality services under any circumstances to be the duty of dispatchers, for which they perceived that continued development of capabilities and the use of previous experiences were a guarantee for quality service provision:

You should build on your own and your colleagues' experiences, and in every case, you have to do the best thing possible. [...] You have to be up-to-date because the first mistake in the dispatch might lead to the death of a patient. [Contributor 3]

\section{Altruism}

From the viewpoint of the participants, under any circumstances, one should not refrain from helping patients, and altruism and empathy help one recognize the patients' problems more substantially.

At nights when operations are overwhelming and tedious, the dispatcher might unconsciously be inclined to dismiss the patient. [...] But any person may have difficulty on the day or the night and need our help. [Contributor 9]

\section{Respect}

According to the participants, respect to the patient brings about the patient and companions' cooperation. Collaboration of the caller and the dispatcher provides a more appropriate ground for obtaining information and making right decisions:

If you treat the people with respect, you will receive their cooperation better.

\section{Impartiality in clinical judgment}

The participants expressed that the dispatchers who do not interfere a patient's social status in clinical judgment and treat all patients fairly and impartially have a better performance.

From the way a patient speaks, it becomes clear what social class $\mathrm{s} /$ he belongs to. [...] It is easier to convince common people so as not to dispatch an ambulance. Nevertheless, our approach to everyone should be equal and respectful.

[Contributor 8]

\section{Professional attitudes}

Identification of the professional attitudes of dispatchers is one of the most important findings of this study. The participants posited that the dispatcher needed to have a set of specific professional attitudes to function appropriately. These attitudes can help better understand the contributors to decision-making in EMD.

\section{Recognition of emergency as any threat to health}

The participants maintained that in contrast to the common presumption, one should not consider only critical patients as emergency patients, and that the condition to dispatch an ambulance does not reside with a serious change in vital signs and the presence of definite and well-documented symptoms in a patient. In fact, any threat to health should be considered an emergency, and the dispatcher should try to discover the potential threats with a curious quest of the patient's condition:

Many cardiac patients have had the preliminary symptoms of ischemia, such as sweating and shortness of breath, long before they were in contact with us, but they had not taken them seriously and thought that an ambulance would not be sent for these simple symptoms. [Contributor 2]

We have had a lot of patients whose initial complaint was a headache or dizziness, and even a feeling of tongue enlargement, but then a stroke was diagnosed. [Contributor 11]

\section{Sensitivity in triage of the patients}

The participants also argued that the dispatcher should examine a patient's medical history with great suspicion 
and sensitivity as if for an unpredictable situation in the EMS. They believed that dispatchers needed, as far as possible, to triage the patient beyond the initial assessment for service so as to avoid any potential change in the patient's condition.

You should not expect that all diagnoses of the dispatcher to be accurate; in contrast, the dispatcher should be pessimistic and skeptical. [...] In general, we need to be pessimistic with the situation and give greater precaution even more than the patient him/herself [...] so that to avoid losing even a single patient. [Contributor 11]

If we do not take the supposedly simple complaints and the mild symptoms of patients seriously, then the patient will most often than not call back with serious conditions. [Contributor 1]

\section{Response to all requests}

According to the participants, the dispatcher needs to take all the calls for assistance seriously and be responsive to all requests, and should provide appropriate assistance for each patient, rather than screen or reject some of the contacts.

I have experienced that there is not even a simple complaint behind which there is not a potential emergency situation. I even had a patient with an aching leg whose final diagnosis was arterial blockage, or a patient with a back pain the cause of which was fracture of the vertebrae due to bone metastasis. Therefore, I understood that all calls must be taken seriously. [...] It is not really possible to reject the patient before evaluating the patient on the site. [Contributor 7]

\section{Care for early warnings}

The participants had found that many diseases, along with known symptoms generally cited at the time of their diagnosis, tend to have a number of simple presentations or early warnings. Therefore, they emphasized that in obtaining a medical history, one should focus on the search for early warnings and that even the common people needed to be aware of these warnings.

Many patients do not tell you the classic symptoms of the disease on the phone or they are still at the onset of the disease and do not have any clear signs. However, each of their simple complaints may turn out to be a warning; for example, a woman who describes her husband to be just sweating or having short breaths, he may be having the first symptoms and warnings of a heart problem and later undergo a chest pain. [Contributor 12]

\section{Attention to the EMS social support responsibility}

According to the participants, social support and the inspiration of confidence and peace of mind in citizens through responsiveness under all circumstances can be considered a vital view of the EMS.

Suppose a soldier serving as a border guard who has an old father living alone at home. As we are not worried about our security thanks to such soldiers, the soldier should also be secure to think that the EMS will be openly accountable to his father in case of need. [Contributor 8]

\section{Commitment to organizational goals and standards}

The participants stated that recognition and obedience of the EMS goals and the professional rules laid down will provide a framework to avoid mistakes and respond optimally to the patients.

Sometimes, colleagues complain after an operation that the case was irrelevant and that they were sent uselessly at the middle of the night. [...] There are always complaints like this. It's important to know the rules and try to do your job well. [Contributor 2]

\section{Professional temperance}

According to the participants, the dispatcher should have peace of mind and professional temperance in dealing with patients notwithstanding the special conditions of the EMD, such as the tense work environment and complicated conditions:

You must do your job in the dispatch despite anything that is happening. Whatever the patient might be like on the phone - either aggressive, upset, or hostile - you should not want to be involved or affected. [Contributor 14]

\section{Responsiveness}

The responsiveness as a main category was formed by aggregating two categories of the codes emerging in the study "personal values and beliefs", which are mainly based on individualistic and internal characteristics of the dispatcher in dealing with patients, and "professional attitudes", which explain the dispatcher's approach to provision of EMS. Responsiveness, as a human factor, seems to oblige the dispatcher to strive for improved quality of services and act as an incentive for timely delivery of services and improved professional performance. 


\section{Discussion}

Based on the findings of the study, the contributors to the timely and appropriate decision-making of dispatchers were identified to be in two categories of values and professional attitudes. Each of the categories seems to have a role in making the right decision. The findings showed that personal values and beliefs are the basis for the dispatcher's decision-making.

Values are part of the structure of professional ethics in the health system. Their identification in our study corresponds with the results of other studies in which the professional code of ethics was introduced as an inseparable principle of clinical decision-making. ${ }^{19}$ It is consistent with studies that underline commitment to values such as impartiality, respect for human rights, treatment of individuals irrespective of their social status, excellence of services, self-sacrifice, and usefulness for others which together make people satisfied with the EMS..$^{20-22}$

Also, according to the findings of this study, the role of faith in the accuracy of the dispatchers' judgments should be taken into account. Faith leads to adherence to this common principle of all religions that whatever one finds improper for him/herself, s/he should find improper for others, which is a golden rule in the professional code of ethics. ${ }^{23}$ Despite the similarity in values in different societies, it is necessary to identify and strengthen these values in each country in accordance with the prevailing social, cultural, economic, and religious conditions in order to identify and apply professional values. ${ }^{24}$

The association between proper decision-making and employee attitudes is one of the findings of this research, which is solely based on the views of the participants. Other studies have also highlighted the relationship between EMS staff attitudes and improved relief delivery. ${ }^{25,26}$

An important finding in this study was the identification of the specific professional attitudes category. It seems that the dispatcher needs to make fundamental changes to his/her mission in order to make a proper decision. A study pointed to the lack of understanding of the philosophy and mission of the EMS in developing countries, stating that the training of dispatchers is effective in institutionalizing the EMS values and understanding its philosophy. ${ }^{12}$ These views are expected to develop as the dispatchers get trained and experienced. As an example, a $45 \%$ rate of reduced response time was reported upon education given to the dispatchers. ${ }^{27}$ Similar studies have also pointed to the role of professional experience in nursing decision-making. ${ }^{19}$

Some of these attitudes have already been described. The importance of having specific professional attitudes for a dispatcher has been highlighted, including accuracy and sensitivity to locate life-threatening cases..$^{28} \mathrm{~A}$ solution to overcome the limited information received about the patient is to overtriage patients so that the patients with mild and atypical symptoms would not be overlooked. Excessive accuracy may lead to loss of patients. In this regard, some studies have reported the dispatcher's undertriage as a reason for the death of patients seeking help..$^{29}$

Also, the need to care for early warnings along with the typical symptoms of diseases should be considered as a professional attitude of the dispatcher. Paying attention to early warnings by dispatchers and also by the general public increases the chances of timely emergency treatment. ${ }^{30,31}$ Accordingly, new strategies have been employed in many countries to train the community of the proper use of EMS in the event of early warnings. ${ }^{32}$

Other professional attitudes identified in this study can also be the basis for improved decision-making on the part of dispatchers. For example, the recognition of emergency as any threat to health can orient attention to patients who have contacted right before their vital signs undergo severe changes and their chance of survival diminishes after the onset of changes. Therefore, the dispatcher's attitude has to be changed and any potential health threat to the patient should be considered an emergency in need of assistance.

Also, response to all requests for help should be included in the standards of the prehospital emergency system, and attention to the social support role of the EMS will orient dispatchers from exploring the emergency to preparing for any help to people at risk. Care given to a lonely, elderly person who may not currently have critical conditions but may be at risk due to inability to take care of him/herself is an example of attention to the supportive role of the EMS. The professional temperance acts as a way to adapt to EMD's tense circumstances and provides the basis for proper decisionmaking in spite of the stressful situations.

It seems that values and beliefs that have an internal and individualistic nature, along with acquirable factors such as professional attitudes, are important in decision-making by dispatchers. When individuals start working in a certain workplace, they bring values and beliefs with them. Nonetheless, professional attitudes arise from training and experiences of working in the prehospital environment.

Based on the findings of this study conducted in the low- to middle-income country of Iran, strengthened responsiveness in dispatchers can be conducive to their improved decision-making. It seems that institutionalization of responsiveness in the EMS system is effective in 
improving many EMS processes. Responsiveness in the health system staff has been described in different studies as commitment, sense of responsibility, and advocacy of patients' rights, ${ }^{33,34}$ and one of the concepts for quality in care delivery. ${ }^{35}$

Given that the World Health Organization (WHO) has ranked the Iranian health system as 100th among world health systems in terms of responsiveness, ${ }^{36}$ it becomes more relevant to recognize the dimensions of responsiveness as introduced in this study, including values and professional attitudes. Studies show that a health system that is socially responsive and accountable receives greater support from the community. ${ }^{37}$ Timely and respectful response has been emphasized in the WHO's framework for assessing responsiveness in the treatment process, where responsiveness has been regarded as a fundamental concept related to human rights, a shared responsibility in the health system, and an entity that can be increased without cost and technology. ${ }^{38}$

It seems that the identified concepts and the relationship between them can help better understand the factors affecting timely and appropriate decision-making by dispatchers. The categories also tend to provide useful information for dispatchers and managers in order to modify the established views. Also, these concepts can be a groundbreaking field for applied research and implementation of interventions to improve the quality of EMD.

To implement responsiveness as the key finding of this study, it is suggested that personal values be included in the recruitment criteria for dispatchers and be strengthened during work. Moreover, the conditions for acquiring professional attitudes can be provided via training and sharing of experiences in order for correct and timely decision-making on the part of dispatchers. Investigation of the ways to strengthen these categories can be the subject of further studies.

\section{Limitations}

This study conducted in the context of Iran can be limited in terms of generalizability to other societies. However, it seems that given the shared problems in low- and middle-income countries, the results can be applicable in these countries. Regarding the focus of the study on the extraction of mental factors influencing decision-making in dispatchers, the effects of other aspects, such as the EMD equipment and structure, have not been investigated. Of course, it seems to be possible to improve dispatcher processes by finding ways to develop individual capabilities without expending on equipment and structures.

\section{Conclusion}

This study identified responsiveness as a main category for improving the decision-making process among dispatchers. Its institutionalization in dispatchers can improve the prompt and proper decision-making. The participants in this research have posited a dispatcher's commitment to personal values and the specific professional attitudes as crucial in order to achieve responsiveness.

\section{Acknowledgment}

This study was a part of a PHD thesis supported financially by Iran University of Medical Sciences (Grant No IUMS/ SHMIS-1393/30).

\section{Disclosure}

The authors report no conflicts of interest in this work.

\section{References}

1. Concannon TW, Griffith JL, Kent DM, et al. Elapsed time in emergency medical services for patients with cardiac complaints: are some patients at greater risk for delay? Circ Cardiovasc Qual Outcomes. 2009;2(1):9-15.

2. Peden M, Scurfield R, Sleet D, et al. World Report on Road Traffic Injury Prevention. Geneva: World Health Organization; 2004.

3. World Health Organization. Global status report on road safety 2013 Available from: http://www.who.int/violence_injury_prevention/ road_safety_status/2013/en/. Accessed November 6, 2015.

4. Robertson-Steel I. "Reforming Emergency Care": the ambulance impact. A personal view. Emerg Med J. 2004;21(2):207-211.

5. Kobusingye OC, Hyder AA, Bishai D, Hicks ER, Mock C, Joshipura M. Emergency medical systems in low- and middle-income countries: recommendations for action. Bull World Health Organ. 2005;83(8):626-631.

6. Naghavi M, Abolhassani F, Pourmalek F, et al. The burden of disease and injury in Iran 2003. Popul Health Metr. 2009;7:9.

7. Modaghegh MH, Roudsari BS, Sajadehchi A. Prehospital trauma care in Tehran: potential areas for improvement. Prehosp Emerg Care. 2002;6(2):218-223.

8. Peyravi M, Khodakarim S, Ortenwall P, Khorram-Manesh A. Does temporary location of ambulances ("fluid deployment") affect response times and patient outcome? Int J Emerg Med. 2015;8(1):37.

9. Bahrami MA, Maleki A, Ranjbar Ezzatabadi M, Askari R, Ahmadi Tehrani GH. Pre-hospital emergency medical services in developing countries: a case study about EMS response time in Yazd, Iran. Iran Red Crescent Med J. 2011;13(10):735-738.

10. Nielsen K, Mock C, Joshipura M, Rubiano AM, Zakariah A, Rivara F. Assessment of the status of prehospital care in 13 low- and middleincome countries. Prehosp Emerg Care. 2012;16(3):381-389.

11. Ek B, Svedlund M. Registered nurses' experiences of their decisionmaking at an emergency medical dispatch centre. J Clin Nurs. 2015;24(7-8):1122-1131.

12. Shah CH, Ismail IM, Mohsin SS. Ambulance response time and emergency medical dispatcher program: a study in Kelantan, Malaysia. Southeast Asian J Trop Med Public Health. 2008;39(6):1150-1154.

13. Blanchard IE, Doig CJ, Hagel BE, et al. Emergency medical services response time and mortality in an urban setting. Prehosp Emerg Care. 2012;16(1):142-151.

14. Khorasani-Zavareh D, Khankeh HR, Mohammadi R, Laflamme L, Bikmoradi A, Haglund BJ. Post-crash management of road traffic injury victims in Iran. Stakeholders' views on current barriers and potential facilitators. BMC Emerg Med. 2009;9:8. 
15. Castren M, Karlsten R, Lippert F, et al; Emergency Medical Dispatch expert group at the Utstein Consensus Symposium 2005. Recommended guidelines for reporting on emergency medical dispatch when conducting research in emergency medicine: the Utstein style. Resuscitation. 2008;79(2):193-197.

16. Peyravi M, Ortenwal P, Djalali A, Khorram-Manesh A. An overview of shiraz emergency medical services, dispatch to treatment. Iran Red Crescent Med J. 2013;15(9):823-828.

17. Braun V, Clarke V. Using thematic analysis in psychology. Qualitative Research in Psychology. 2006;3(2):77-101.

18. Vaismoradi M, Turunen H, Bondas T. Content analysis and thematic analysis: implications for conducting a qualitative descriptive study. Nurs Health Sci. 2013;15(3):398-405.

19. Pouralizadeh M, Khankeh H, Ebadi A, Dalvandi A. Factors influencing nursing students' clinical judgment: a qualitative directed content analysis in an Iranian context. J Clin Diagn Res. 2017;11(5):JC01-JC04.

20. Ebrahimian A, Seyedin H, Jamshidi-Orak R, Masoumi G. Exploring factors affecting emergency medical services staffs' decision about transporting medical patients to medical facilities. Emerg Med Int. 2014;2014:215329.

21. Pang D, Senaratana W, Kunaviktikul W, Klunklin A, McElmurry BJ. Nursing values in China: the expectations of registered nurses. Nurs Health Sci. 2009;11(3):312-317.

22. Jensen A, Lidell E. The influence of conscience in nursing. Nurs Ethics. 2009;16(1):31-42.

23. Childress JF, Beauchamp TL. Principles of Biomedical Ethics. USA: Oxford University Press; 2001.

24. Shahriari M, Mohammadi E, Abbaszadeh A, Bahrami M. Nursing ethical values and definitions: a literature review. Iran J Nurs Midwifery Res. 2013;18(1):1-8.

25. Citerio G, Galli D, Pesenti A. Early stroke care in Italy - a steep way ahead: an observational study. Emerg Med J. 2006;23(8):608-611.

26. Kleindorfer DO, Lindsell CJ, Broderick JP, et al. Community socioeconomic status and prehospital times in acute stroke and transient ischemic attack: do poorer patients have longer delays from 911 call to the emergency department? Stroke. 2006;37(6):1508-1513.
27. Hisamuddin NA, Hamzah MS, Holliman CJ. Prehospital emergency medical services in Malaysia. J Emerg Med. 2007;32(4):415-421.

28. Jia J, Band R, Abboud ME, et al. Accuracy of emergency medical services dispatcher and crew diagnosis of stroke in clinical practice. Front Neurol. 2017;8:466.

29. Andersen MS, Johnsen SP, Hansen AE, et al. Preventable deaths following emergency medical dispatch — an audit study. Scand J Trauma Resusc Emerg Med. 2014;22:74.

30. Kleindorfer D, Khoury J, Broderick JP, et al. Temporal trends in public awareness of stroke: warning signs, risk factors, and treatment. Stroke. 2009;40(7):2502-2506.

31. Jauch EC, Cucchiara B, Adeoye O, et al. Part 11: adult stroke: 2010 American Heart Association Guidelines for Cardiopulmonary Resuscitation and Emergency Cardiovascular Care. Circulation. 2010;122(18 Suppl 3):S818-S828.

32. Fessler SJ, Simon HK, Yancey AH, 2nd, Colman M, Hirsh DA. How well do General EMS 911 dispatch protocols predict ED resource utilization for pediatric patients? Am J Emerg Med. 2014;32(3):199-202.

33. Verpeet E, Dierckx de Casterle B, Van der Arend A, Gastmans CA. Nurses' views on ethical codes: a focus group study. J Adv Nurs. 2005;51(2):188-195.

34. Wros PL, Doutrich D, Izumi S. Ethical concerns: comparison of values from two cultures. Nurs Health Sci. 2004;6(2):131-140.

35. Schluter J, Seaton P, Chaboyer W. Understanding nursing scope of practice: a qualitative study. Int J Nurs Stud. 2011;48(10): $1211-1222$

36. World Health Organization. The world health report 2000 - Health systems: improving performance. Available from: http://www.who.int/ whr/2000/en/. Accessed November 6, 2015

37. McClellan M, Udayakumar K, Thoumi A, et al. Improving care and lowering costs: evidence and lessons from a global analysis of accountable care reforms. Health Aff (Millwood). 2017;36(11):1920-1927.

38. de Silva A. A framework for measuring responsiveness. GPE discussion paper series: no. 32. Available from: www.portal.pmnch.org/responsiveness/papers/paper32.pdf. Accessed November 15, 2015.
Journal of Multidisciplinary Healthcare

\section{Publish your work in this journal}

The Journal of Multidisciplinary Healthcare is an international, peerreviewed open-access journal that aims to represent and publish research in healthcare areas delivered by practitioners of different disciplines. This includes studies and reviews conducted by multidisciplinary teams as well as research which evaluates the results or conduct of such teams or health

\section{Dovepress}

care processes in general. The journal covers a very wide range of areas and welcomes submissions from practitioners at all levels, from all over the world The manuscript management system is completely online and includes a very quick and fair peer-review system. Visit http://www.dovepress.com/ testimonials.php to read real quotes from published authors. 